\title{
Correlates of Burnout in Small Independent Primary Care Practices in an Urban Setting
}

\author{
Batel Blechter, MA, Nan Jiang, PhD, Charles Cleland, PhD, Carolyn Berry, PhD, \\ Olugbenga Ogedegbe, MD, MS, MPH, FACP, and Donna Shelley, MD, MPH
}

Background: Little is known about the prevalence and correlates of burnout among providers who work in small independent primary care practices ( $<5$ providers).

Methods: We conducted a cross-sectional analysis by using data collected from 235 providers practicing in $\mathbf{1 7 4}$ small independent primary care practices in New York City.

Results: The rate of provider-reported burnout was $13.5 \%$. Using bivariate logistic regression, we found higher adaptive reserve scores were associated with lower odds of burnout (odds ratio, 0.12 ; 95\% CI, 0.02-0.85; $P=.034)$.

Conclusion: The burnout rate was relatively low among our sample of providers compared with previous surveys that focused primarily on larger practices. The independence and autonomy providers have in these small practices may provide some protection against symptoms of burnout. In addition, the relationship between adaptive reserve and lower rates of burnout point toward potential interventions for reducing burnout that include strengthening primary care practices' learning and development capacity. (J Am Board Fam Med 2018;31:529-536.)

Keywords: Cross-Sectional Analysis, Logistic Regression, New York City, Prevalence, Primary Health Care, Professional Burnout

Physician burnout is a major concern for the health care industry. In 2011, a national survey reported that $45.5 \%$ of US physicians were experiencing symptoms of burnout. ${ }^{1}$ By 2014 , the rate had increased to $54.4 \% .^{1}$ Burnout is associated with low job satisfaction and reduced productivity among physicians and may negatively impact quality of care. $^{2,3}$

Several studies have examined the individuallevel and organizational-level correlates of burnout. $^{4-6}$ A study of family doctors from 16 European countries found that high rates of burnout, as as-

This article was externally peer reviewed.

Submitted 1 September 2017; revised 21 December 2017; accepted 31 December 2017.

From Bloomberg School of Public Health, Johns Hopkins University, Baltimore, MD (BB); School of Medicine, New York University, New York, NY (NJ, CB, OO, DS); and Meyers College of Nursing, New York University, New York (CC).

Funding: This project was supported by Agency for Healthcare Research and Quality (AHRQ 1R18HS023922-01).

Conflict of interest: none declared.

Corresponding author: Donna Shelley, MD, MPH, School of Medicine, New York University, New York, NY 10016 (E-mail: Donna.Shelley@nyumc.org). sessed using the Maslach Burnout Inventory Survey, were associated with intention to change jobs, substance and tobacco use, younger age, and male sex. ${ }^{4}$ A 2013 meta-analysis of physician surveys conducted in the United States and Europe found that for US physicians, lower rates of burnout were associated with greater perceived autonomy, a quality and safety culture at work, effective coping skills, and less work/life conflict. ${ }^{5}$

Research on physician burnout has focused primarily on hospital settings or large primary care practices. We are not aware of any studies of burnout that have included large numbers of small independent primary care practices (SIPs). Although the number of SIPs ( $<5$ providers) in the United States has been decreasing, they continue to serve a significant proportion of the population. ${ }^{7-9}$ Yet, burnout among providers in these important sources of primary care is not well characterized. This article aimed to fill this research gap by assessing the rate of burnout and factors associated with burnout among providers practicing in SIPs located in New York City (NYC). 


\section{Methods}

\section{Data Source}

This study analyzed data collected as part of the HealthyHearts NYC (HHNYC) trial that is evaluating the impact of external practice facilitation on the adoption of clinical guidelines for cardiovascular disease prevention and treatment in SIPs. ${ }^{10}$ The project is funded through the Agency for Health Care and Quality's EvidenceNOW initiative and was approved by the New York University School of Medicine Institutional Review Board. ${ }^{11}$ Details about the HHNYC study design have been described in a previous article. ${ }^{10}$ Study sites that are participating in HHNYC are members of a practice network created and managed by the NYC Department of Health and Mental Hygiene's Primary Care Information Project. ${ }^{12}$ This analysis used data from baseline surveys collected from 235 providers in 174 SIPs.

\section{Measures}

Provider burnout was assessed with a single item measure that has been validated against the Maslach Burnout Inventory: "Using your own definition of burnout, please indicate which of the following statements best describes how you feel about your situation at work?"13 Answer options included: "I enjoy my work. I have no symptoms of burnout," "Occasionally I am under stress, and I do not always have as much energy as I once did, but I do not feel burned out," "I am definitely burning out and have 1 or more symptoms of burnout, such as physical and emotional exhaustion," "The symptoms of burnout that I am experiencing will not go away. I think about frustrations at work a lot," and "I feel completely burned out and often wonder if I can go on practicing. I am at the point where I may need some changes." Consistent with previous studies $^{14}$, respondents were categorized as burned out if they checked 1 of the last 3 options.

Practice characteristics included the number of providers, characterized as "solo provider" and "2 or more providers"; medically underserved area designation (yes/no); patient-centered medical home $(\mathrm{PCMH})$ status (yes/no); adaptive reserve; Change Process Capacity Questionnaire (CPCQ); patient panel size; and patient race/ethnicity (see Appendix). The CPCQ is a measure of practices' strategies for quality improvement (eg, our center uses periodic measurement of care quality). ${ }^{15}$ The adaptive re- serve measure assesses the nature of leadership (eg, supports change, shared vs authoritarian), communication practices, trust and teamwork, collective efficacy, and culture of learning. ${ }^{16,17}$

Both CPCQ and adaptive reserve include 14 items answered with a 5-point Likert scale from "strongly disagree" (1 point) to "strongly agree" (5 points). Consistent with the literature, we converted the value of each individual item from a 1 to 5 to a 0 to 1 scale, and then calculated the mean score by averaging the values of nonmissing items. ${ }^{16}$ Thus, the scores range from 0 to 1 . A larger value indicates an organization's greater ability to implement change and a higher level of adaptive reserve. PCMH status, patient panel size, and patient race/ethnicity were obtained from the Primary Care Information Project's internal administrative database. The medically underserved area designation was obtained from the US Department of Health and Human Services' Health Resources and Services Administration website. ${ }^{18}$

Provider characteristics included the number of hours the provider works per week in the participating practice site and the number of years he/she has worked in the practice.

\section{Statistical Analysis}

Descriptive statistics summarized practice and provider characteristics. About $7 \%$ of all data were missing. To address missing data, we used multiple imputation with the "mice" package of the R statistical computing environment. ${ }^{19-21}$ A total of 20 imputed datasets were generated, with bivariate regression analyses repeated for each imputation. We used the "pan" package to accommodate the multilevel missing data (practice and provider). ${ }^{22}$ Generalized estimating equations ${ }^{23}$ were used to estimate bivariate associations between independent variables and burnout. All significance tests were 2-tailed. $\mathrm{R}$ version 3.4.1 was used for data analyses.

\section{Results}

\section{Characteristics of SIPs and Providers}

Table 1 shows the characteristics of SIPs and providers. Most (66.9\%) SIPs were solo provider practices and $46.5 \%$ were PCMH recognized. Of the providers, 204 were physicians (MDs) and 31 were nurse practitioners/physician's assistants and $13.5 \%$ reported burnout. 
Table 1. Characteristics of Small Independent Primary Care Practices and Providers

\begin{tabular}{|c|c|c|c|c|}
\hline & & & $\mathrm{n}$ & $(\%)$ \\
\hline \multirow[t]{15}{*}{ Practice characteristics $(\mathrm{N}=174)$} & \multirow{10}{*}{$\begin{array}{l}\text { Categorical } \\
\text { variables }\end{array}$} & Number of providers & & \\
\hline & & Solo provider & 113 & $(66.9 \%)$ \\
\hline & & $\geq 2$ providers & 56 & $(33.1 \%)$ \\
\hline & & MUA designation* & & \\
\hline & & Yes & 75 & $(43.1 \%)$ \\
\hline & & No & 99 & $(56.9 \%)$ \\
\hline & & PCMH recognition & & \\
\hline & & Yes & 81 & $(46.5 \%)$ \\
\hline & & No & 93 & $(53.5 \%)$ \\
\hline & & & Mean & $\underline{(\mathrm{SD})}$ \\
\hline & \multirow{5}{*}{$\frac{\text { Continuous }}{\underline{\text { variables }}}$} & CPCQ & 0.78 & $(0.19)$ \\
\hline & & Patient panel size & 2207 & $(2252)$ \\
\hline & & $\begin{array}{l}\% \text { of non-Hispanic white } \\
\text { patients }\end{array}$ & 19 & $(26.8)$ \\
\hline & & Adaptive reserve & 0.78 & $(0.17)$ \\
\hline & & & $\mathrm{n}$ & $(\%)$ \\
\hline \multirow[t]{6}{*}{ Provider characteristics $(N=235)$} & \multirow{4}{*}{$\begin{array}{l}\text { Categorical } \\
\text { variable }\end{array}$} & Burnout & & \\
\hline & & Yes & 29 & $(13.5 \%)$ \\
\hline & & No & 185 & $(86.5 \%)$ \\
\hline & & & Mean & $(\mathrm{SD})$ \\
\hline & \multirow{2}{*}{$\begin{array}{l}\text { Continuous } \\
\text { variables }\end{array}$} & Working hours per week & 36.9 & $(13.4)$ \\
\hline & & Years in practice & 12.4 & $(8.2)$ \\
\hline
\end{tabular}

*Data extracted from Health Resources and Service Administration website.

MUA, medically underserved area; PCMH, patient-centered medical home; CPCQ, Change Process Capability Questionnaire; SD, standard deviation.

\section{Factors Associated with Provider Burnout}

Bivariate analysis (Table 2) showed that a higher adaptive reserve score was associated with lower

Table 2. Bivariate Analysis of the Factors Associated with Provider Burnout

\begin{tabular}{lcc}
\hline & OR (95\% CI) & $P$ \\
\hline Practice characteristics (N = 174) & & \\
Number of providers (solo) & $0.88(0.39-1.97)$ & .752 \\
MUA designation* (yes) & $0.56(0.25-1.30)$ & .177 \\
PMCH recognition (yes) & $1.44(0.64-3.25)$ & .379 \\
CPCQ & $0.12(0.01-1.16)$ & .067 \\
Patient panel size & $1.04(0.70-1.55)$ & .854 \\
\% of non-Hispanic white patients & $1.01(0.99-1.02)$ & .287 \\
Adaptive reserve & $0.12(0.02-0.85)$ & .034 \\
Provider characteristics (N =235) & & \\
Working hours per week & $1.02(0.99-1.04)$ & .220 \\
Years in practice & $0.99(0.95-1.05)$ & .825 \\
\hline
\end{tabular}

*Data extracted from Health Resources and Service Administration website

CI, confidential interval; OR, odds ratio; MUA, Medically underserved area; PCMH, patient-centered medical home; CPCQ, Change Process Capability Questionnaire. odds of burnout (odds ratio, $0.12 ; 95 \%$ CI, $0.02-$ $0.85 ; P=.034)$. Other variables were not associated with burnout. Since only 1 variable was associated with provider burnout, we did not undertake multivariate analyses. Bivariate associations were similar under pairwise deletion of missing data and multiple imputation.

\section{Discussion}

We found a remarkably low burnout rate $(13.5 \%)$ among providers practicing in SIPs in NYC compared with the burnout rate among physicians in previous studies. ${ }^{1,14}$ Almost $70 \%$ of the SIPs in this study were solo practices. Therefore, one explanation for this finding could be the autonomy (ie, control of work environment) associated with owning one's own practice as opposed to working in an integrated health system or Federally Qualified Health Center where providers are subject to greater administrative regulations. ${ }^{24}$ Studies have found an association between low work control or autonomy and higher levels of burnout and that autonomy varies by practice size, with smaller practices 
reporting greater logistic autonomy than larger practices. 5,6,14,24,25 Compared with larger practices, SIPs may have deeper relationships with their patients, which may lead to greater job satisfaction and less burnout among providers. ${ }^{26}$

The number of hours providers reported working per week was lower than in previous reports. For example, the Physician Worklife Study reported a mean of 54.6 hours worked per week among US physicians. ${ }^{27}$ However, the way we asked this question may have underestimated working hours, because it assessed only those hours worked at the study site. In addition, in contrast to previous studies, we found no correlation between hours worked and burnout. ${ }^{4}$ This concept may be better captured by measuring work-life balance, which has also been strongly associated with burnout. ${ }^{28}$ Further study is needed to better characterize the association between work-life balance, hours worked, and burnout among providers working in SIPs.

We found that higher adaptive reserve scores were associated with lower levels of burnout. The National Demonstration Project, which studied implementation of $\mathrm{PCMH}$ in family practices, found that sites that were more successful in transforming their practices had greater "internal capacity for organizational learning and development," which the authors defined as adaptive reserve. ${ }^{17}$ The National Demonstration Project did not examine the relationship between adaptive reserve and burnout, but previous studies reported a link between burnout and a range of work environment characteristics that are captured in the adaptive reserve measure. ${ }^{6,14,29,30}$

The relationship between adaptive reserve and provider burnout suggests that interventions to reduce burnout in primary care practices should focus on strengthening factors that support organizational capacity for change (ie, strong communication, leadership supports, innovation). These factors may manifest differently in SIPs as compared with larger systems but may be just as important in influencing provider burnout.

The study has several limitations. First, data were collected from SIPs in NYC. Therefore, the findings may not be generalized to providers who work in these settings outside of NYC. Second, we conducted multiple imputations to fill missing values when alternative data sources were unavailable. The process may reduce the statistical power.
Third, the EvidenceNOW studies were focused primarily on site-level outcomes and, therefore, surveys included a wide range of practice characteristics, but they largely excluded provider demographic characteristics that may have offered additional insights into the findings. ${ }^{31}$

Despite these limitations, this study adds new information about factors that may impact burnout among providers practicing in SIPs. Future research is needed to better define the complex relationships between individual and organizational factors, including adaptive reserve and provider burnout and how these factors impact patient outcomes in SIPs.

To see this article online, please go to: http://jabfm.org/content/ 31/4/529. full.

\section{References}

1. Shanafelt TD, Hasan O, Dyrbye LN, et al. Changes in burnout and satisfaction with work-life balance in physicians and the general US working population between 2011 and 2014. Mayo Clin Proc 2015;90: 1600-13.

2. Dewa CS, Loong D, Bonato S, Thanh NX, Jacobs P. How does burnout affect physician productivity? A systematic literature review. BMC Health Serv Res 2014; 14:325.

3. Shanafelt TD, Balch CM, Bechamps G, et al. Burnout and medical errors among American surgeons. Ann Surg 2010;251:995-1000.

4. Soler JK, Yaman H, Esteva M, et al. Burnout in European family doctors: the EGPRN study. Fam Pract 2008;25:245-65.

5. Lee RT, Seo B, Hladkyj S, Lovell BL, Schwartzmann L. Correlates of physician burnout across regions and specialties: a meta-analysis. Hum Resour Health 2013;11:48.

6. Rabatin J, Williams E, Baier Manwell L, Schwartz MD, Brown RL, Linzer M. Predictors and outcomes of burnout in primary care physicians. J Prim Care Community Health 2016;7:41-3.

7. Hing E, Uddin S. Visits to primary care delivery sites: United States, 2008. NCHS Data Brief: No. 47. Hyattsville, MD: U.S. Department of Health and Human Services, Centers for Disease Control and Prevention, National Center for Health Statistics; 2010.

8. Hing E, Schappert SM. Generalist and Specialty Physicians: Supply and Access, 2009-2010. NCHS Data Brief: No. 105. Hyattsville, MD: U.S. Department of Health and Human Services, Centers for Disease Control and Prevention, National Center for Health Statistics; 2012.

9. Liaw WR, Jetty A, Petterson SM, Peterson LE, Bazemore AW. Solo and small practices: a vital, 
diverse part of primary care. Ann Fam Med 2016;14: 8-15.

10. Shelley DR, Ogedegbe G, Anane S, et al. Testing the use of practice facilitation in a cluster randomized stepped-wedge design trial to improve adherence to cardiovascular disease prevention guidelines: HealthyHearts NYC. Implement Sci 2016;11:88.

11. U.S. Department of Health \& Human Services Agency for Healthcare Research and Quality. EvidenceNOW: advancing heart health in primary care. Available from: https://www.ahrq.gov/evidencenow/ index.html. Accessed August 20, 2017.

12. City of New York. Primary Care Information Project. 2016; Available from: http://www1.nyc.gov/site/ doh/providers/electronic-records.page. Accessed April 20, 2017.

13. Rohland BM, Kruse GR, Rohrer JE. Validation of a single-item measure of burnout against the Maslach Burnout Inventory among physicians. Stress Health 2004;20:75-9.

14. Linzer M, Manwell LB, Williams ES, et al. Working conditions in primary care: physician reactions and care quality. Ann Intern Med 2009;151:28-36.

15. Solberg LI, Asche SE, Margolis KL, Whitebird RR. Measuring an organization's ability to manage change: the change process capability questionnaire and its use for improving depression care. Am J Med Qual 2008; 23:193-200.

16. Jaén CR, Crabtree BF, Palmer RF, et al. Methods for evaluating practice change toward a patient-centered medical home. Ann Fam Med 2010;8:S9-20.

17. Nutting PA, CrabTree BF, McDaniel RR. Small primary care practices face four hurdles - including a physician-centric mind-set-in becoming medical homes. Health Aff (Millwood) 2012;31:2417-22.

18. U.S. Department of Health \& Human Services Health Resources \& Services Administration. Medically underserved areas and populations (MUA/ Ps). Available from: https://bhw.hrsa.gov/shortagedesignation/muap. Accessed April 20, 2017.

19. van Buuren S, Groothuis-Oudshoorn K. mice: multivariate imputation by chained equations in R. J Stat Softw 2011;45:1-67.

20. van Buuren S. Flexible imputation of missing data. Boca Raton, FL: Chapman and Hall/CRC; 2012.
21. R Core Team. R: a language and environment for statistical computing. R Foundation for Statistical Computing, Vienna, Austria. 2017; Available from: https://www.R-project.org/. Accessed August 22, 2017.

22. Schafer JL, Zhao JH. pan: multiple imputation for multivariate panel or clustered data ( $\mathrm{R}$ package version 1.4). 2016; Available from: https://cran.rproject.org/web/packages/pan/index.html. Accessed August 20, 2017.

23. Hardin JW, Hilbe JM. Generalized Estimating Equations. 2nd ed. Boca Raton, FL: Chapman and Hall/CRC; 2012.

24. Lin KY. Physicians' perceptions of autonomy across practice types: is autonomy in solo practice a myth? Soc Sci Med 2014;100:21-9.

25. Gregory ST, Menser T. Burnout among primary care physicians: a test of the areas of worklife model. J Healthc Manag 2015;60:133-48.

26. Casalino LP, Pesko MF, Ryan AM, et al. Small primary care physician practices have low rates of preventable hospital admissions. Health Aff (Millwood) 2014;33:1680-8.

27. Linzer M, Visser MRM, Oort FJ, et al. Predicting and preventing physician burnout: results from the United States and the Netherlands. Am J Med 2001; 111:170-5.

28. Sinsky CA, Dyrbye LN, West CP, Satele D, Tutty M, Shanafelt TD. Professional satisfaction and the career plans of US physicians. Mayo Clin Proc 2017; 92:1625-35.

29. Panagioti M, Panagopoulou E, Bower P, et al. Controlled interventions to reduce burnout in physicians: a systematic review and meta-analysis. JAMA Intern Med 2017;177:195-205.

30. Linzer M, Poplau S, Grossman E, et al. A cluster randomized trial of interventions to improve work conditions and clinician burnout in primary care: results from the Healthy Work Place (HWP) study. J Gen Intern Med 2015;30:1105-11.

31. Cohen DJ, Balasubramanian JA, Gordon L, et al. A national evaluation of a dissemination and implementation initiative to enhance primary care practice capacity and improve cardiovascular disease care: the ESCALATES study protocol. Implement Sci 2016; 11:86. 
Appendix A. Measures of Independent Variables

\begin{tabular}{lll}
\hline Variable (Data Source) & Survey Item & Coding Method \\
\hline
\end{tabular}

\section{Practice characteristics}

Number of provider (practice survey)

MUA designation (HRSA website)

PCMH recognition (PCIP)

Patient panel size (PCIP)

$\%$ of non-Hispanic white patients (PCIP)

Adaptive reserve (practice member survey)
Which of the following best describes your practice site size?

A. Solo practice

B. 2 to 5 clinicians (medical doctor, doctor of osteopathic medicine, nurse practitioner, physician's assistant)

C. 6 to 10 clinicians

D. 11 to 15 clinicians

E. 16 or more clinicians

Has your practice site been designated as a MUA or medically underserved population by the HRSA?

A. Yes B. No

Is your practice site recognized or accredited as a PCMH?

A. Yes B. No

Please estimate the average patient panel size for a full-time clinician in your practice site.

Please give the percentage of your patients in the following categories.

A. White

B. Black/African American

C. American Indian or Alaska Native

D. Asian

E. Native Hawaiian or other Pacific Islander

F. Some Other Race/Mixed Race

G. Percent unknown

1. Mistakes have led to positive changes here

2. I have many opportunities to grow in my work

3. People in our practice actively seek new ways to improve how we do things

4. People at all levels in this office openly talk about what is and isn't working

5. Leadership strongly supports practice change efforts

6. After trying something new, we take time to think about how it worked

7. Most of the people who work in our practice seem to enjoy their work

8. It is hard to get things to change in our practice

9. This practice is a place of joy and hope

10. This practice learns from its mistakes

11. Practice leadership promotes an environment that is an enjoyable place to work

12. People in this practice operate as a real team

13. When we experience a problem in the practice, we make a serious effort to figure out what's really going on

14. Leadership in this practice creates an environment where things can be accomplished

Each item is offered a 5-point Likert scale as below.

A. Strongly disagree

B. Disagree

C. Neutral

D. Agree

E. Strongly agree
$1=$ "Solo provider" (Answer option A) $0=$ " $\geq 2$ providers" (Other than answer option A)

$1=$ "Yes" $0=$ "No

$1=$ "Yes" $0=$ "No"

Each item was scored on a 1 ("strongly disagree") to 5 ("strongly agree") scale.

To obtain the mean adaptive reserve score, we converted the score for each item to a 0 to 1 scale and then summed up all the nonmissing values and divided by the total number of nonmissing items. 
Appendix A. Continued

\begin{tabular}{|c|c|c|}
\hline Variable (Data Source) & Survey Item & Coding Method \\
\hline CPCQ (practice survey) & $\begin{array}{l}\text { Indicate the extent to which you agree or disagree that your } \\
\text { practice site has used the following strategies to improve } \\
\text { cardiovascular preventive care: } \\
\text { 1. Providing information and skills training } \\
\text { 2. Using opinion leaders, role modeling. Or other vehicles to } \\
\text { encourage support for change } \\
\text { 3. Changing or creating systems in the practice that make it } \\
\text { easier to provide high quality care } \\
\text { 4. Removal or reduction of barriers to better quality of care } \\
\text { 5. Using teams focused on accomplishing the change process }\end{array}$ & $\begin{array}{l}\text { Each item was scored on a } 1 \\
\text { ("strongly disagree") to } 5 \\
\text { ("strongly agree") scale. } \\
\text { To obtain the mean } \\
\text { CPCQ score, we } \\
\text { converted the score for } \\
\text { each item to a } 0 \text { to } 1 \text { scale, } \\
\text { and then summed up all } \\
\text { the nonmissing values and } \\
\text { divided by the total } \\
\text { number of nonmissing } \\
\text { items. }\end{array}$ \\
\hline
\end{tabular}

6. Delegating to nonclinician staff the responsibility to carry out aspects of care that are normally the responsibility of physicians

7. Providing to those who are charged with implementing improved care the power to authorize and make the desired changes

8. Periodic measurements of care quality for assessing compliance with any new approach to care

9. Reporting measurements of practice performance on cardiovascular disease prevention measures (such as aspirin for patients at risk for ischemic vascular disease) for compassion with their peers

10. Setting goals and benchmarking rates of performance quality on cardiovascular disease prevention measures at least yearly

11. Customizing the implementation of cardiovascular disease prevention care changes to the practice

12. Using rapid cycling, piloting, pretesting, or other vehicles for reducing the risk of negative results for introducing organization-wide change in care

13. Deliberately designing care improvements so as to make clinician participation less work than before

14. Deliberately designing care improvements to make the care process more beneficial to the patient

Each item is offered a 5-point Likert scale as below.

A. Strongly disagree

B. Disagree

C. Neutral

D. Agree

E. Strongly agree

F. NA

Provider characteristics

Hours worked per week (Practice Member Survey)

Years in practice (Practice Member Survey)

Burnout (Practice Member Survey)
How many hours per week do you work at this practice site?

How many years have you worked in this practice site?

Using your own definition of burnout, please indicate which of the following statements best describes how you feel about your situation at work

A. I enjoy my work. I have no symptoms of burnout

B. Occasionally I am under stress, and I don't always have as much energy as I once did, but I don't feel burned out

C. I am definitely burning out and have one or more symptoms of burnout, such as physical and emotional exhaustion
1 = "Burnout" (Answer options C-E) $0=$ "Not burned out" (Answer options A or B) 


\begin{tabular}{ll}
\hline Variable (Data Source) & \multicolumn{1}{c}{ Survey Item } \\
\hline D. The symptoms of burnout that I'm experiencing won't go \\
away. I think about frustrations at work a lot \\
$\begin{array}{l}\text { E. I feel completely burned out and often wonder if I can go } \\
\text { on practicing. I am at the point where I may need some } \\
\text { changes }\end{array}$ \\
\hline
\end{tabular}

MUA: medically underserved area, PCMH, patient-centered medical home; CPCQ, Change Process Capability Questionnaire; HRSA, Health Resources and Service Administration; PCIP, Primary Care Incentive Payment Program. 